\section{Indicadores de morbimortalidade hospitalar de tuberculose no Município de São Paulo}

\author{
Indicators of tuberculosis \\ morbimortality in hospitals in the \\ city of São Paulo, Brazil
}

Vera Maria Neder Galesi ${ }^{1}$

Margarida Maria Mattos Brito de Almeida²

'Divisão de tuberculose - CVE-CCD - Secretaria de Estado da Saúde de São Paulo

${ }^{2}$ Departamento de Epidemiologia, Faculdade de Saúde Pública, Universidade de São Paulo

Correspondência: Vera Maria Neder Galesi. Rua Souza Noschese, 43 - São Paulo,SP. CEP 04726-200. E-mail: veragalesi@uol.com.br

\section{Resumo}

Introdução: O tratamento de tuberculose é realizado atualmente nos serviços ambulatoriais, ficando a internação recomendada para os casos de maior gravidade ou com problemas sociais. No Município de São Paulo, contudo ainda tem sido internado um número expressivo de doentes de tuberculose. Objetivo do trabalho: Estudar as características sociodemográficas e clínicoepidemiológicas dos doentes internados com tuberculose, residentes no Município de São Paulo em 2001. Metodologia: Utilizando como fonte de dados o sistema de informação em tuberculose do Estado, (EPI TB) programa de registro, acompanhamento e análise de notificações e dados de população do Deinfo/ SEMPLA e FIPE, foram calculados indicadores de morbimortalidade segundo variáveis existentes nas fichas de notificação dos doentes internados. Resultados e Discussão: No município de S.Paulo, no ano de 2001, foram internados 2.473 doentes com tuberculose. $\mathrm{O}$ coeficiente de internação desses doentes foi de 23,5 casos por 100.000 habitantes, o coeficiente de mortalidade encontrado foi de 4,1 por 100.000 habitantes, tendo ocorrido 485 óbitos e sendo a taxa de letalidade de $17,4 \%$. A taxa de coinfecção Tb/HIV foi de $32,7 \%$ entre os internados e de $12,5 \%$ entre os não internados, indicando que a epidemia de Aids exerceu forte influência tanto na magnitude quanto na gravidade da situação desses doentes, sendo a maior letalidade $(48,4 \%)$ a da forma disseminada/miliar, com a maioria de casos em doentes HIV positivos. Entretanto, se excluirmos os casos dos portadores de HIV, a taxa de letalidade ainda se mantém alta, $15,1 \%$, mostrando também a gravidade da tuberculose. Conclusões: Os resultados mostraram indicadores com valores preocupantes, ficando evidente a importância do acompanhamento dos mesmos para monitorar a situação da tuberculose. Recomendações: As vigilâncias epidemiológicas municipais devem estabelecer fluxos e estratégias de acompanhamento dos doentes internados com tuberculose principalmente nos grandes centros urbanos, para garantir a continuidade do tratamento e modificar o cenário encontrado.

Palavras-chave: Tuberculose. Internação. Indicadores de morbimortalidade hospitalar. 


\section{Abstract}

Introduction: The treatment of tuberculosis is currently carried out in outpatient health services and hospitalization is only recommended for the more severe cases or for those facing social problems. In São Paulo, however, a considerable number of tuberculosis patients are still hospitalized. Objective: To study the socio-demographic and clinical-epidemiological characteristics of hospitalized tuberculosis patients in the city of São Paulo in 2001. Specific Objetive: To calculate tuberculosis morbimortality indicators for inpatients. Methods: Indicators of morbimortality of inpatients were calculated taking as sources, data from the São Paulo state tuberculosis information system - (Epitb) registration program, and the follow-up and analysis of reports and population data from Deinfo/ SEMPLA and FIPE. Results: A total of 2,473 tuberculosis patients were hospitalized in the city of São Paulo in 2001. The hospitalization rate for these patients was 23.5 per 100,000 inhabitants, and the mortality rate found was 4.1 per 100,000 inhabitants with 485 deaths, yielding a fatality rate of $17.4 \%$. Discussion: The TB/HIV coinfection rate was of $32.7 \%$ among inpatients and $12.5 \%$ among those not hospitalized, showing that the Aids epidemic has caused a considerable impact both in the magnitude and in the severity of the situation of hospitalized tuberculosis patients. The higher fatality rate $(48.4 \%)$ is of disseminated / miliary tuberculosis, of which the majority occurs in HIV-positive patients. If, however, known HIV-positive cases are excluded, the fatality rate still remains high, i.e. $15 \%$, showing that the severity of cases is not only due to co-infection. Conclusions: Taking all points into consideration, the indicators showed an alarming situation. Recommendation: It is recommended that the epidemiological surveillance of municipalities establish flows and follow-up strategies for tuberculosis patients who have been hospitalized, mainly those in large urban centers, in order to guarantee continuity of care and modify the present scenario.

Keywords: Tuberculosis. Hospitalization. Indicators of hospital morbidity and mortality.

\section{Introdução}

O tratamento da tuberculose atualmente é feito nos serviços ambulatoriais, ficando a internação para os casos com maior gravidade ou com problemas sociais.

Até o século XIX eram feitas as mais variadas conjeturas sobre a natureza e a causa da tuberculose. Da mesma forma a história de seu tratamento foi a mais exótica e empírica enquanto durou o desconhecimento sobre sua origem.

O movimento sanatorial (de sanitorium - sanare - palavra latina para curar), baseado na recomendação de ar puro e dieta teve início no século XIX, expandindo-se após a descoberta do bacilo por Koch, que confirmou ser a doença infecciosa e contagiosa, e por isso o isolamento dos doentes foi considerado importante medida para controlar a doença.

No Estado de São Paulo, na década de 40 , semelhante ao que ocorria no mundo, a estratégia prioritária para o tratamento era a hospitalização, havendo 3.741 leitos disponíveis para internação em 1947 e 8.067 em $1950^{1}$.

A partir de 1956, inúmeros estudos passam a evidenciar as vantagens do tratamento ambulatorial em relação à hospitalização, sendo o mais conhecido o estudo de Madras, realizado na Índia ${ }^{2}$. Este estudo mostrou que a conversão bacteriológica e radiológica, bem como o risco de infecção para os contactos no grupo de internados, quando comparado ao dos não internados, foi semelhante. Assim, concluiu que além da desvantagem social do sanatório, separando os doentes da família, causando ruptura nos laços familiares, essa medida não causava impacto na diminuição da transmissão familiar da doença ${ }^{3}$. Iniciou-se então no mundo um movimento pela deshospitalização, assimilado gradualmente pelo nosso país.

Em 1980 houve uma reorganização do atendimento da tuberculose no Estado e foi criado um sistema centralizado de controle de vagas. Este sistema conseguiu adequar as internações e reduzi-las. Consolidou-se a política de tratamento ambulatorial. 
No Município de São Paulo, o número de casos descobertos por ano é expressivo, representando o maior contingente de casos em números absolutos do Estado de São Paulo. Em 2001 foram descobertos 6.523 casos novos, com um coeficiente de incidência de 58,3 por 100.000 habitantes e um coeficiente de mortalidade por tuberculose causa básica de 4,6 por 100.000 habitantes, valores superiores às taxas do Estado que neste ano foram de 47,1 para a incidência e 3,1 para a mortalidade. Em 2001, 58\% dos casos do Município foram descobertos em hospitais/pronto socorros. Existem nesse Município cerca de 40 hospitais gerais que internam mais de 10 casos de tuberculose por ano. Além disso, dois hospitais conveniados do SUS em Campos do Jordão, com cerca de 400 leitos de longa permanência, representam a infraestrutura hospitalar que oferece retaguarda ao Município para internação de moradores de rua ou albergados, alcoolistas e outros casos sociais. Apesar do DOT (tratamento diretamente observado) ser a política adotada no Estado para o tratamento dos doentes, o hospital ainda representa importante papel no controle da tuberculose nos grandes centros urbanos.

O objetivo geral deste trabalho foi estudar as características sociodemográficas e clínico-epidemiológicas dos doentes internados com tuberculose, residentes no Município de São Paulo em 2001. O objetivo específico foi calcular por meio das variáveis encontradas, indicadores de morbimortalidade para os doentes internados com tuberculose.

\section{Método}

Utilizou-se como fonte de dados o sistema de informação em tuberculose do Estado de São Paulo (EpiTb), programa de registro, acompanhamento e análise de notificações. Este sistema consolida as variáveis contidas na Ficha de notificação de tuberculose, que inclui dados demográficos, situação de maior risco de adoecimento (moradores em situação de rua e portadores de HIV), clínicos (forma clínica da doença), hospital onde ocorreu a internação e o desfecho do tratamento, que pode incluir o óbito. A partir de casos notificados em 2001 de residentes no Município de São Paulo, foi construído um banco de dados no Epi Info 6.0 e foram calculados indicadores de morbimortalidade para os doentes internados. Os dados de população foram obtidos de estimativas realizadas pela Secretaria Municipal de Planejamento Urbano de São Paulo (SEMPLA), com base nos dados dos Censos Demográficos de 1991 e 2000 do Instituto Brasileiro de Geografia e Estatística (IBGE) e da Fundação Instituto de Pesquisas Econômicas (FIPE) para a estimativa do número de moradores em situação de rua.

\section{Resultados}

No Município de São Paulo, em 2001, foram internados com tuberculose 2.473 doentes, de um total de 6.600 casos diagnosticados como tuberculose e residentes neste Município.

A distribuição dos casos segundo faixa etária mostrou que a maior proporção de internados $(50,7 \%)$ foi a de 30 a 49 anos para ambos os sexos, faixa esta que representa também a população mais atingida pela doença. Os homens internados apresentaram média de idade $(40,1)$ superior a dos não internados $(36,2)$, sendo que entre as mulheres não houve diferença significativa (35,5 e 35,8). Entre as mulheres, a proporção de internados menores de 30 anos $(38,7 \%)$ foi o dobro daquela encontrada entre os homens $(17,5 \%)$.

O coeficiente de internação com tuberculose desses doentes foi de 23,5 casos por 100.000 habitantes para todas as formas clínicas e de 18 por 100.000 para a forma pulmonar. O coeficiente de mortalidade hospitalar segundo o total de menções de tuberculose encontrado foi de 4,1 por 100.000 habitantes, tendo ocorrido 485 óbitos.

Este coeficiente se apresentou com valores mais elevados entre os homens $(6,2$ por 100.000 habitantes) do que entre as 
mulheres $(2,1)$. O maior coeficiente de mortalidade hospitalar ocorreu para os homens na faixa de 50 anos e mais $(12,1)$.

A taxa de letalidade dos casos de tuberculose internados foi de $17,4 \%$.

Cerca de $47,4 \%$ dos óbitos foram casos coinfectados de Tb/HIV, mostrando a importância da Aids para explicar esta alta letalidade. A forma clínica mais grave entre os internados foi a miliar/disseminada com taxa de letalidade de $41,2 \%$.

Na Figura 1 pode-se observar os três indicadores segundo faixa etária, sendo que o coeficiente de mortalidade e a taxa de letalidade se apresentaram com maior valor entre os maiores de 50 anos e o coeficiente de internação para aqueles entre 20 e 49 anos de idade.

Em relação aos moradores em situação de rua, os indicadores de morbimortalidade são ainda mais preocupantes. Adoeceram por tuberculose e foram identificados no sistema de informação como moradores em situação de rua em 2001, no Município de São Paulo, 115 pessoas, tendo sido internados 111. O coeficiente de internação foi de $1.197,5$ por 100.000 habitantes, o coeficiente de mortalidade foi de 366,8 por 100.000 habitantes e a taxa de letalidade de $30,6 \%$, conforme se pode visualizar na Figura 2.

A distribuição da internação segundo hospital, mostrou entre as 2.320 internações nas quais os hospitais foram identificados (em 153 internações não foi possível identificar o hospital), que $47,5 \%$ ocorreram em 78 hospitais gerais, $21,1 \%$ em três hospitais de referência (Instituto de Infectologia Emílio Ribas, Hospital do Mandaqui e Centro de Referência e Treinamento DST/Aids), 9,9 \% nos três hospitais de longa permanência e os restantes $2,3 \%$ em outros hospitais conforme mostra a Figura 3.

\section{Discussão}

A distribuição segundo idade e sexo encontrada neste estudo foi bastante semelhante a encontrada em outros estudos nacionais e internacionais ${ }^{4-8}$, indicando que a distribuição desigual de casos de tuberculose em relação a estas variáveis ocorre em todo o mundo desenvolvido e em desenvolvimento, sendo ainda obscuras as razões para explicar este fato.

O Programa Nacional de Controle da

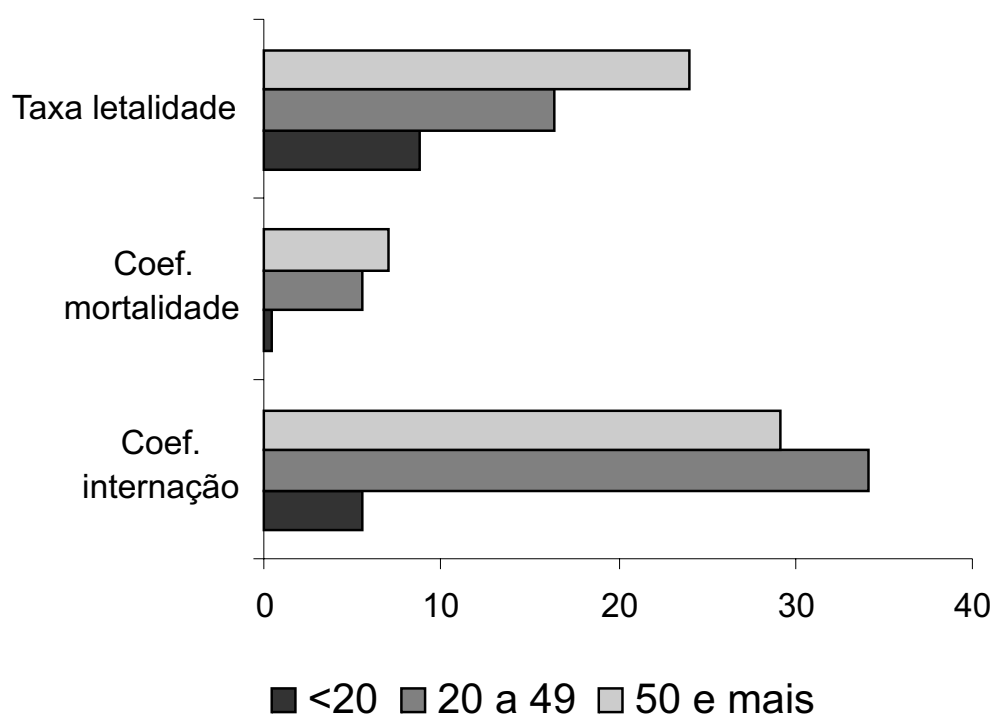

Figura 1 - Coeficiente* de internação e de mortalidade e Taxa** de letalidade para os doentes internados com tuberculose segundo faixa etária, no Município de São Paulo, 2001.

Figure 1 - Hospitalization and mortality rates* and fatality rate ${ }^{* *}$ for patients hospitalized for tuberculosis according to age group, in the City of São Paulo, 2001.

* por 100.000 habitantes $/{ }^{* *}$ por 100 habitantes 


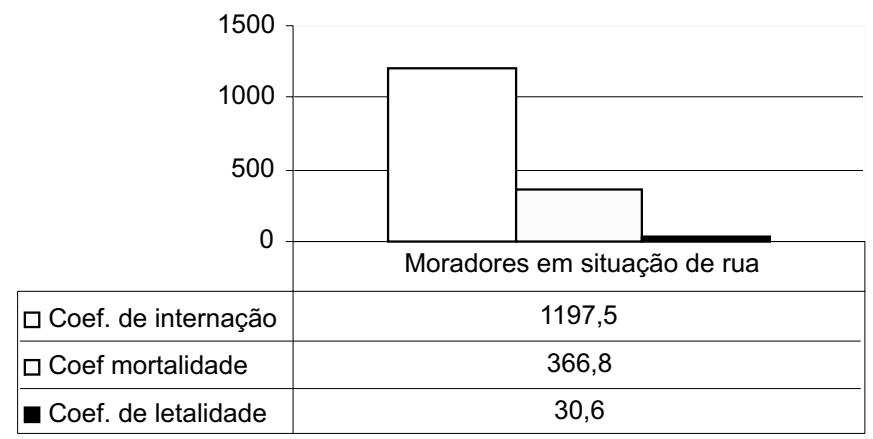

Figura 2 - Coeficiente* de internação e de mortalidade e Taxa** de letalidade para os doentes internados internados com tuberculose, moradores em situação de rua no Município de São Paulo, 2001.

Figure 2 - Hospitalization and mortality rates* and fatality rate ${ }^{* *}$ for homeless patients hospitalized due to tuberculosis, in the City of São Paulo, 2001.

* por 100.000 habitantes / ** por 100 habitantes

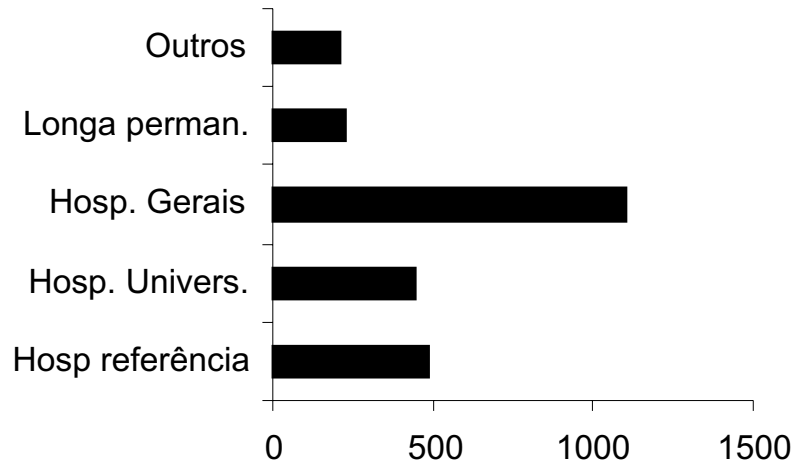

Figura 3 - Casos internados segundo tipo de hospital, Município de São Paulo, 2001. Figure 3 - Cases hospitalized according to type of hospital, City of São Paulo, 2001.

Tuberculose, em 1984, estimava a necessidade de internação em $10 \%$ dos casos descobertos ${ }^{1}$, e mesmo nessa época o Estado de São Paulo internava $26,1 \%$ dos casos notificados (4.559/17.449), ou seja, muito acima do preconizado. A maior disponibilidade de leitos no Estado de São Paulo pode ter sido um fator para explicar as maiores taxas de internação.

Um parâmetro para este indicador encontrado na literatura nacional foi descrito por Silva Júnior em $1998^{9}$, referindo para o Estado de São Paulo um coeficiente de internação de tuberculose pulmonar de 11 por 100.000 habitantes, construído com dados do Sistema de Informações Hospitalares do SUS (SIH), cujo objetivo é o pagamento de serviços pelos prestadores ao SUS. Como se pode observar, este coeficiente obtido para o Estado foi bastante in- ferior ao encontrado para o Município em 2001. Explicações para este fato vão desde diferenças existentes entre os registros dos dois sistemas de informação até a possibilidade do agravamento da situação de internação no Município de 1998 a 2001. Independente do que tenha causado a diferença, é importante ressaltar a necessidade de interligar os dois sistemas melhorando a qualidade da informação e possibilitando uma utilização mais adequada dos indicadores gerados pelos mesmos.

O coeficiente de internação parece ser um bom indicador para o acompanhamento da morbimortalidade hospitalar.

Com relação aos óbitos ainda permanece também pouco clara a razão para as desigualdades relacionadas ao gênero entendendo como tal "não apenas as diferenças fenotípicas entre os sexos, mas também a 
variedade de comportamentos, expectativas e papéis que existem num contexto socioeconômico cultural" ${ }^{10}$. Dolin ${ }^{11}$, em 1998, questiona se o fato de haver maior número de casos entre os homens diz respeito a diferenças biológicas ou de gênero, concluindo que permanecem ainda obscuras as razões das diferenças entre os sexos ${ }^{10,11}$.

A taxa de letalidade encontrada foi bastante elevada. A comparação desta taxa com a de dois agravos agudos considerados de alta letalidade como a meningite meningocócica e a leptospirose no Estado de São Paulo, em 2001, que foram de respectivamente 18,9\% (com 228 óbitos) e 14,1\% (com 105 óbitos) ${ }^{12,13}$, confirma que a letalidade dos casos internados com tuberculose é alta e semelhante a de agravos agudos.

É efetivamente preocupante uma taxa de letalidade de $17,5 \%$ para todas as formas clínicas, chegando a atingir $66,1 \%$ na forma miliar, e sendo mais elevada do que a de muitas doenças agudas. A taxa de coinfecção Tb/HIV encontrada foi de $32,7 \%$ entre os internados e de $12,5 \%$ entre os não internados, indicando que a epidemia de Aids exerceu forte influência tanto na magnitude quanto na gravidade da situação dos doentes internados com tu- berculose. Entretanto, se excluirmos os casos HIV+, a taxa de letalidade ainda se mantém alta, $15,1 \%$, mostrando que a gravidade dos casos está relacionada com a própria tuberculose, conforme se pode observar na Figura 4.

Os moradores em situação de rua são sabidamente de maior risco de adoecimento e de internação com tuberculose. Segundo Mrks $^{14}$ e cols., avaliando uma coorte de doentes de tuberculose em situação de rua nos Estados Unidos (2000), possíveis razões para explicar as maiores taxas de internação foram severidade da doença, intolerância aos medicamentos devido ao uso de álcool e drogas, a decisão de quem atendeu o caso de assegurar adesão, ou até mesmo em razão de o doente não ter onde morar. Nesse estudo foi constatado que $50 \%$ dos casos eram alcoolistas. Nakamura e cols. (1999) ${ }^{15}$ estudando em Tóquio, de 1992 a 1996, 1.938 moradores em situação de rua descrevem numa análise de co-morbidades uma associação entre psicose alcoólica, síndrome de dependência de álcool e tuberculose pulmonar.

Comparativamente em São Paulo, no período do estudo, 57,7\% deles eram alcoolistas e $2,7 \%$ tinham referência de doença mental.

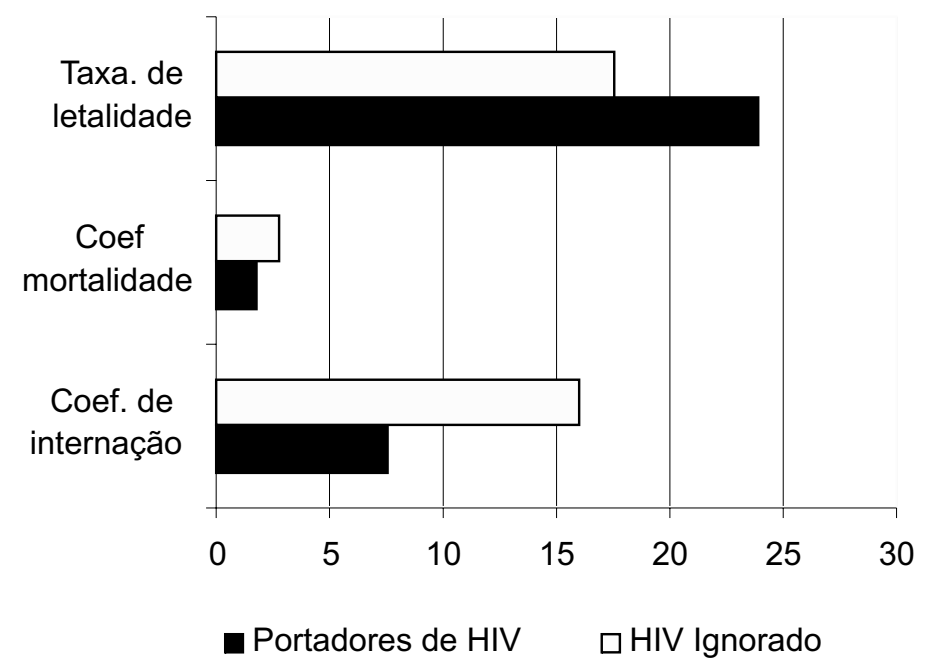

Figura 4 - Coeficiente* de internação e de mortalidade e Taxa** de letalidade para os doentes internados com tuberculose segundo resultado de HIV no Município de São Paulo, 2001.

Figure 4 - Hospitalization and mortality rates* and fatality rate** for patients hospitalized due to tuberculosis according to HIV status in the City of São Paulo, 2001.

* por 100.000 habitantes / ** por 100 habitantes 
Quanto ao tipo de hospital que internou o caso, os hospitais gerais são os que mais internam, sendo que $10 \%$ das internações ocorreram nos leitos de longa permanência, reservados aos casos com necessidade sociais.

\section{Conclusões}

Os valores encontrados para os indicadores de morbimortalidade hospitalar de doentes internados com tuberculose no município de São Paulo, em 2001, são bastante elevados, expressando uma situação grave.

Apesar das altas taxas de coinfecção, a gravidade dos casos não está relacionada apenas a esta comorbidade, significando provavelmente diagnóstico tardio da tuberculose.

\section{Recomendações}

Apesar das internações por tuberculose terem sido necessárias, a gravidade dos indicadores apontam para a necessidade de investimento em diagnóstico precoce na porta de entrada do sistema. Sabendo que $58 \%$ dos casos no ano de 2001 no $\mathrm{Mu}$ nicípio de São Paulo foram descobertos nas emergências de hospitais e pronto-socorros, parecem ser estes os locais prioritários para se investir na agilidade e qualidade do diagnóstico precoce, bem como na orientação adequada para o tratamento.

Portanto devem ser instituídos protocolos de atendimento de forma a identificar precocemente os suspeitos, garantir retaguarda de exames bacteriológicos com resultados rápidos, implementando-se também medidas de biosegurança. São diretrizes do Estado de São Paulo garantir o resultado de baciloscopia em quatro horas nas urgências e 24 horas na rede ambulatorial.

Além disso, torna-se necessário instituir fluxos de encaminhamento dos casos descobertos nas emergências, de forma a garantir a continuidade do tratamento nas unidades básicas de saúde.

\section{Referências}

1. Nogueira PA. Internações por tuberculose no Estado de São Paulo 1984 a 1997. [Tese de Livre-Docência] São Paulo: Faculdade de Saúde Pública da USP; 2001.

2. World Health Organization. A current comparison of home and sanatorium treatment of pulmonary tuberculosis in South Îndia. Bull WHO 1959; 21(1).

3. Toman K. Tuberculosis: case-finding and chemotherapy. Geneva: World Health Organization; 1979.

4. Fandinho FCO, Kritski AL, Hofer C, Conde Jr H, Ferreira FMC, Silva MG Fonseca LS. Drug Resistence Patterns among Hospitalized Tuberculous Patients in Rio de Janeiro, Brazil, 1993-1994. Mem Inst Oswaldo Cruz 1999; 94(4): 543-7.

5. Rabahi MF, Rodrigues AB, Queiroz de MF, de Almeida Netto JC, Kritsky AL. Non complience with tuberculosis treatment by patients at a tuberculosis and AIDS reference hospital in midwestern Brazil. Braz J Infect Dis 2002; 6(2): 63-73.

6. Albuquerque MFM, Leitão CCS, Campelo ARL, Souza W, Salustiano A. Fatores prognósticos para um desfecho do tratamento da tuberculose pulmonar em Recife, Pernambuco, Brasil. Rev Panam Salud Publica 2001; 9(6): 368-74.
7. Henn L, Nagel F, Dal Pizzo F. Comparison between human immunodeficiency virus positive and negative patients with tuberculosis in Southern Brazil. Mem Inst Oswaldo Cruz 1999; 94(3); 377-81.

8. Singleton LRN, Turner M, Haskal R, Etkind S, Tricarico M, Nardel E. Long term hospitalization for tuberculosis control. JAMA 1997; 278(10): 838-42.

9. Silva Junior JB, Mendes ACG, Campos Neta TJ, Lyra TM, Medeiros KR, Sá DA. Sistema de informações hospitalares - Fonte complementar na Vigilância e monitoramento de doenças transmitidas entre pessoas. Informe Epidemiológico do SUS 2000; 9(2): 137-62.

10. Thorson A, Diwan VK. Background to gender and tuberculosis workshop: Tuberculosis - A global Public health problem. NHVReport 1998: 3.

11. Dolin P. Tuberculosis Epidemiology from a gender perspective. NHVReport 1998: 3.

12. São Paulo (Estado). Secretaria de Estado da Saúde. Centro de Vigilância Epidemiológica "Prof. Alexandre Vranjac"; julho de 2005. disponível em http:// www.cve.saude.sp.gov.br/htm/ resp/ dm9004_ofeta.htm. Acessado em 16/05/2005. 
13. São Paulo (Estado). Secretaria de Estado da Saúde. Centro de Vigilância Epidemiológica "Prof. Alexandre Vranjac"; Março de 2004. Disponível em: http://

www.cve.saude.sp.gov.br/htm/zoo/LEPTO_ANO8601.htm

14. Marks SM, Taylor Z, Burrows NR, Qayad MG, Miller B. Hospitalization of homeless persons with tuberculosis in the United States. Am J of Public Health 2000, 90(3): 435-8.
15. Nakamura K, Takeuchi S, Watanabe M. Disease patterns of the homeless in Tokyo. J Urban Health 1999; 76(1): 7384

Recebido em: 17/02/06 Versão final reapresentada em: 06/11/06 aprovado em: 20/12/06 